\title{
EVALUACIÓN ECONÓMICA DEL DAÑO MORAL EN EL ÁMBITO DE LOS ACCIDENTES DE TRABAJO: UN MÉTODO BASADO EN INDICADORES ESTADÍSTICOS NORMALIZADOS
}

\author{
JOSÉ LUIS NAVARRO ESPIGARES (*) \\ JOSÉ AURELIANO MARTÍN SEGURA (**)
}

(*) Universidad de Granada, Hospital Universitario Virgen de las Nieves

(**) Universidad de Granada, UNED.

\section{RESUMEN}

Dos recientes pronunciamientos de la Sala $4^{\mathrm{a}}$ de lo Social del Tribunal Supremo han puesto fin a la doctrina jurisprudencial por la que, para evitar el "enriquecimiento injusto" del trabajador accidentado, se exoneraba al empresario del deber de reparar los perjuicios causados en un accidente de trabajo o enfermedad profesional, por el incumplimientos de sus obligaciones en materia de seguridad. Este cambio jurisprudencial obliga a abordar la cuantificación económica de los distintos componentes indemnizatorios. En este trabajo ofrecemos un novedoso método para la evaluación económica directa del daño moral basado en los años de vida ajustados por calidad perdidos por los trabajadores que sufren un accidente de trabajo. El método propuesto se ofrece como una alternativa ventajosa frente a la utilización del baremo de tráfico.

\section{PALABRAS CLAVES}

Daño moral, indemnización para trabajadores, valor de la vida, salud laboral, legislación laboral.

\begin{abstract}
Two recent Social Supreme Court rulings have put an end to the jurisprudential doctrine that prevented the "unfair enrichment" of an injured worker. This doctrine exempted the employer from the obligation of repairing the damage caused by work accidents or occupational diseases, stemming from breaches of the employer's security obligations. The jurisprudential change requires addressing the economic quantification of the various components of workers' compensation. Our study offers a novel method for assessing the economic value of moral damage based on Quality Adjusted Life Years lost by workers who suffer a work accident. The proposed method is presented as an alternative that is more appropriate than the use of the traffic scale.
\end{abstract}

\section{KEY WORDS}

Moral damage, workers' compensation, value of life, occupational health, legislation labour 


\section{INTRODUCCIÓN}

Recientemente se han producido dos importantes pronunciamientos de la Sala $4^{\text {a }}$ de lo Social del Tribunal Supremo (TS) en Sala General, concretamente las Sentencias en Unificación de Doctrina de 17 de julio de 2007, que vienen a poner fin a la doctrina jurisprudencial por la que, para evitar el "enriquecimiento injusto" del trabajador accidentado, se exoneraba al empresario del deber de reparar los perjuicios causados en un accidente de trabajo o enfermedad profesional, por el incumplimientos de sus obligaciones en materia de seguridad, al descontarse del total de la indemnización lo percibido por el trabajador como prestaciones de la Seguridad Social, lo que venía a suponer a sensu contrario, un enriquecimiento también injusto del empresario culpable.

Esta jurisprudencia ha sido matizada por el propio TS en su sentencia de 2 de octubre de 2007 en la que se descarta el descuento de las prestaciones de la Seguridad Social si se ha utilizado el baremo de tráfico.

Como se expone en uno de los fallos, el problema surge debido a que son factibles dos soluciones para abordarlo. La primera, denominada técnica de la suplementariedad o de acumulación absoluta, que es la que sigue la Sala Primera de lo Civil del Tribunal Supremo, consistente en considerar que las prestaciones de seguridad social y la acción civil de responsabilidad protegen al trabajador accidentado de forma autónoma e independiente, por lo que son acumulables; y la segunda, denominada de complementariedad, o de acumulación relativa, que es la que sigue la Sala Cuarta de lo Social del mismo Tribunal, y que considera que ambas prestaciones, la de la seguridad social y la indemnización por responsabilidad civil, responden a una misma finalidad y no pueden aplicarse con total independencia.

La novedad de estos dos fallos radica en que cambian el criterio de la Sala Cuarta respecto a la forma de practicar el descuento por las cantidades percibidas por el trabajador accidentado de la Seguridad Social, pues se entiende que estas prestaciones sólo compensan al trabajador del lucro cesante que le provoca la pérdida de salarios, por lo que habrá de ser de este único concepto del que se deduzcan, y no del resto de componentes de la indemnización, como el daño corporal (lesiones físicas y psíquicas), el daño moral (sufrimiento psíquico o espiritual) y el daño emergente (pérdida patrimonial directamente vinculada con el hecho dañoso). Por tanto, para que esta doctrina pueda aplicarse es necesario que en las demandas que se planteen se desglosen con claridad todos los conceptos indemnizatorios con arreglo a estas reglas.

Además, a pesar de reconocer que en el sistema de indemnización de los accidentes de tráfico no se aplica el principio de culpabilidad, el Tribunal Supremo admite como sistema de valoración el baremo de cuantificación de los daños fijado para los accidentes de tráfico, que se estableció en la Disposición Adicional Octava de la Ley 30/1995, y que hoy se regula en el Real Decreto Legislativo 8/2004, de 29 de Octubre del Texto Refundido de la Ley sobre Responsabilidad Civil y Seguro en la Circulación de Vehículos de Motor (en adelante LRCSCVM). El hecho de que no exista un baremo específico para este tipo de indemnizaciones les lleva a aceptarlo como válido, aunque admiten que, de forma razonada, se puedan aplicar otros criterios de baremación del daño para el cálculo de la indemnización.

Como consecuencia de los cambios comentados en la jurisprudencia, ha surgido la necesidad de establecer una forma de cálculo que permita la cuantificación independiente de cada uno de los conceptos indemnizatorios que surgen a raíz de un accidente de trabajo. En el presente trabajo se abordan las cuestiones relativas a la indemnización para cubrir los daños derivados de los accidentes de trabajo y enfermedades profesionales cuando medie culpa o negligencia del empresario en la adopción de medidas de seguridad. El método propuesto se basa en la utilización combinada del baremo de los accidentes de tráfico, junto con otros criterios de valoración novedosos en este ámbito de aplicación. Tal es el caso de los "años de vida ajustados por calidad, $A V A C$ ", concepto habitualmente empleado en la evaluación de tecnologías sanitarias, y que se utiliza en este estudio para la estimación de la indemnización correspondiente al daño moral. Como veremos más adelante, el valor monetario de un AVAC se establece a partir de criterios totalmente independientes, basados en la disposición a pagar de la sociedad, y desvinculados de los intereses del obligado al pago de la indemnización. Se puede decir, en este sentido, que el método incorpora una perspectiva social sin vinculación alguna con el punto de vista del obligado al pago.

En los dos epígrafes siguientes analizaremos desde una perspectiva legal el concepto de daño moral y los recientes cambios acaecidos en la jurisprudencia social española. Seguidamente, se presenta la metodología propuesta en este trabajo. Esta metodología establece la secuencia de cálculo de la 
indemnización a la que tendría derecho el trabajador accidentado por el concepto de daño moral. Está basada en el valor monetario de los años de vida ajustados por calidad que pierde el trabajador tras sufrir un accidente. Finalmente, las conclusiones enfatizan las ventajas que ofrece el método propuesto así como la oportunidad de su incorporación en la práctica jurídica del ámbito social.

\section{EL CONCEPTO DE DAÑO MORAL EN EL DERECHO DE DAÑOS}

Como explica la profesora Rodríguez Marín en López García, et al. (2007) (1), el daño o perjuicio, junto con la acción u omisión negligente y con el nexo causal, es uno de los presupuestos de la responsabilidad civil aquiliana reconocida en el art. 1902 del Código Civil. De esta forma últimamente se identifica a la responsabilidad extracontractual con el Derecho de Daños. Pero el Daño aparece tanto en la esfera de los daños patrimoniales como en la de los no patrimoniales.

Sin embargo, el Código Civil no proporciona un concepto de daño, por lo que ha tenido que ser la Doctrina la encargada de dar su definición. Así, según LARENZ (1) daño sería "todo menoscabo que a consecuencia de un acaecimiento o evento determinado sufre una persona ya en sus bienes vitales o naturales, ya en su propiedad o en su Patrimonio", o también "toda disminución de la utilidad del individuo dañado"

El daño no debe ajustarse únicamente a ser un Derecho subjetivo, sino que es suficiente con que la lesión se produzca en cualquier interés o atributo de la personal, sea o no patrimonial. Surge así la cuestión de las clases de daños, que tendrían una doble clasificación: daños patrimoniales y extrapatrimoniales.

Los daños patrimoniales serían el daño emergente y el lucro cesante, ambos relacionados con el principio de la reparación integra del daño y con el artículo 1106 del Código Civil(1), aunque esto suponga trasladar la normativa contractual al campo extracontractual.

Así, con el daño emergente trataríamos de averiguar el coste de la reparación y los gastos en los que se incurre con ocasión de daño. Con el lucro cesante se busca indemnizar con la ganancia dejada de obtener, o con las pérdidas de ingresos, que conforme se explica en la STS de 5 de noviembre de 1998 (RJ 1998\8404), referida por la profesora Rodríguez Marín (1), tiene una significación distinta de los daños materiales.

Como daños extrapatrimoniales aparecen el Daño Moral y el Daño Corporal, conceptos ambos difíciles de precisar y sobre los que hay abundante doctrina y jurisprudencia.

El Daño Moral podría definirse, siguiendo a Álvarez Vigaray (1966)(2), por oposición al daño patrimonial, como "aquel perjuicio que no implica una pérdida de dinero, que no entraña para la víctima ninguna consecuencia pecuniaria o disminución de su patrimonio", o también como "aquel daño que no consiste en una pérdida económica o en una falta de ganancia".

A juicio del profesor Álvarez Vigaray, el concepto de daños morales no debe reducirse solamente a los dolores o sufrimientos injustamente ocasionados, sino que en él ha de incluirse todo perjuicio no pecuniario producido por la lesión de un bien de la persona (salud, libertad, honestidad, honor, etc.) o de sus sentimientos y afectos más importantes y elevados. Su nota fundamental sería su naturaleza no patrimonial, que lleva como consecuencia que no puedan evaluarse en dinero, por lo que al no ser posible determinar la cuantía de la indemnización en virtud de una prueba que demuestre la magnitud exacta del daño, ha de ser fijada equitativamente por el juez. Por tanto, en esta definición de Daño Moral de la Doctrina se incluyen claramente los daños en la persona física.

En jurisprudencia más reciente, la STS de 31 de mayo de 2000 (RJ 2000\5089), considera al daño moral como el daño que recae sobre el acervo espiritual de la persona. En la misma línea, la profesora Rodríguez Marín (1) los define como aquellos que recaen en bienes y derechos cuya naturaleza no es patrimonial y por lo tanto carecen de posibilidad de ser reparados en sentido estricto, al no ser susceptibles de tráfico jurídico, como ocurre con el honor, el dolor, la integridad corporal, la tristeza, o la muerte de un ser querido. Por esta razón, continúa, su reparación sólo podrá realizarse de modo aproximado, siendo los usos sociales los que marquen la pauta de valor. Aquí es donde se centra la problemática de estos daños. Lo importante será, por tanto, buscar criterios para cuantificarlos.

(1) "La indemnización de daños y perjuicios comprende, no sólo el valor de la pérdida que hayan sufrido, sino también el de la ganancia que haya dejado de obtener el acreedor....". 
El siguiente problema que se aborda en la Doctrina es el referido a la reparación del daño. En este sentido, el profesor Álvarez Vigaray (2) indica la necesidad de establecer límites a la reparación del daño moral, pues ésta no puede ser absoluta o general, ya que no todo sufrimiento o contrariedad merece que se le conceda indemnización. Más recientemente, la STS de 22 de diciembre de 1990 (RJ 1990\756), establecía que “....según reiterada doctrina de esta Sala, la cuantificación de los daños y perjuicios cuando consisten en menoscabos corporales...no se halla sujeta a previsión normativa alguna, sino que ha de efectuarla el órgano jurisdiccional de la instancia de modo discrecional..". Además, la STC de 13 de junio de 1986 (RTC 1986178), establece la necesidad de detallar en las sentencias las partidas indemnizatorias por las cuales se repara el daño, así como de motivar las cantidades concedidas para evitar el fenómeno de una valoración global. Línea en la que se enmarca la reciente jurisprudencia del TS que se citaba al principio.

Un aspecto interesante respecto a la reparación íntegra del daño es la aceptación de que las indemnizaciones son siempre aproximadas, nunca exactas, pero que admiten el método estadístico para ser fijadas. Así, la STS de 15 de octubre de 1990 (RJ 1990\8126), que avalaría nuestra propuesta fijada más adelante para cuantificar el daño moral, plantea:

“...En este orden de reflexiones conviene recordar que los métodos estadísticos, con base en la técnica matemática, revisten hoy en día una notable perfección y llegan a resultados de una exactitud sorprendente por virtud de la llamada «Ley de los grandes números». Permiten por tanto la posibilidad de llegar a conclusiones correctas a partir de una prueba incompleta. Esa precisión que se consigue incluso mediante el muestreo, sobre un panel a escala muy reducida, como ocurre en las encuestas preelectorales y de mercado, puede llegar a ser total cuando el estudio y la investigación se proyecta sobre un colectivo completo...".

“...Por otra parte, nuestro ordenamiento jurídico viene acogiendo progresivamente estos criterios. En el caso de los daños morales la dificultad de la prueba lleva con frecuencia a éste al Supremo Tribunal a presumir su existencia y la imposibilidad de utilizar parámetros o módulos objetivos le conduce también a valorarlos en una cifra razonable, valoración que obviamente tiene un componente fundamentalmente subjetivo -Sentencias de 13 de diciembre 1979 (RJ 1979|4726) y 2 febrero 1980 (RJ 19801743)-. Lo mismo ocurre con el premio de afec- ción fijado arbitrariamente "ex lege» en un tipo porcentual (5\%). El legislador utiliza, a su vez, métodos estadísticos para la cuantificación de derechos de perfil económico difuso, como la nuda propiedad o el usufructo, y otros varios para los impuestos sobre el patrimonio o sobre transmisiones patrimoniales, mediante técnicas actuariales donde se juega con las expectativas de vida, en virtud de estimaciones probabilísticas que, sin embargo, funcionan como exactas en un colectivo suficientemente amplio...".

Una última cuestión sería la referente a la comprobación y liquidación del Daño Moral. En este sentido resultan clarificadoras las conclusiones del profesor Álvarez Vigaray al indicar que para que los tribunales puedan condenar a la reparación del daño moral hace falta que el actor demuestre su existencia. Se trataría de la prueba del daño moral.

Si el daño resulta consecuencia necesaria de la lesión de determinados derechos subjetivos, no será necesario demostrar directamente que se ha producido el daño moral, pues quedaría probado implícitamente con el hecho lesivo. Tal es el caso de la pérdida de la vida, o de las lesiones en la salud e integridad de las personas, como estamos analizando en el presente trabajo. Pero si se trata de otro tipo de daños, como las calumnias, no se podrá establecer una presunción de la existencia del daño moral, salvo que se produzcan los hechos capaces de producirlo, es decir, de una presunción iudicis, al no estar establecida por la Ley.

Una vez probada la existencia del daño moral, habría que probar su cuantía y alcance, para que el Juez pueda fijarla con arreglo a un criterio de equidad.

\section{LA NUEVA DOCTRINA DE LA SALA DE LO SOCIAL DEL TRIBUNAL SUPREMO}

La nueva jurisprudencia del Tribunal Supremo parte de que, si bien los artículos 123.3 y 127.3 de la LGSS y el 42.3 de la LPRL admiten la compatibilidad entre prestaciones de la Seguridad Social y la indemnización por los daños y perjuicios en los supuestos de accidente de trabajo, sin embargo no existe una regulación legal para calcular su montante.

De la misma forma reconocen que nuestra doctrina es unánime a la hora de mantener el derecho a la reparación íntegra, como manifestación del principio general deducible de los artículos 1101 y 1902 del Código Civil, que obliga a todo el que causa un daño a repararlo, aunque en el ámbito laboral falte la 
norma legal expresa que baremice los daños. Ahora bien, dicha reparación íntegra ha de ser adecuada, proporcionada y suficiente, pero no pasar de ahí, pues en este caso se produciría un enriquecimiento injusto del accidentado, que también está prohibido por el artículo 1.4 del Código Civil al establecer que nadie podrá enriquecerse torticeramente a costa de otro. Y además el cálculo de la indemnización ha de hacerse teniendo en cuenta:

- la naturaleza de los hechos,

- el grado de culpabilidad,

- la dependencia económica,

- las sumas ya percibidas y

- los criterios legales que puedan servir de referencia.

Pero a la hora de concretar los daños es necesario distinguir las categorías básicas de los distintos componentes de los mismos. Así, tendríamos un daño corporal, referido a la integridad física, que cubriría el daño biológico o fisiológico, las lesiones físicas y psíquicas; un daño moral que abarca las consecuencias personales del mismo, el sufrimiento psíquico o espiritual; un daño patrimonial, que se divide en daño emergente, que contempla la pérdida patrimonial directamente vinculada con el hecho dañoso; y un lucro cesante, en el que se incluirían la pérdida de ingresos y de expectativas laborales. Con estas precisiones, a juicio del Tribunal Supremo, se da satisfacción al principio I.3 de la Resolución 757 del Comité de Ministros del Consejo de Europa (14 de marzo de 1975), expresivo de que «en la medida de lo posible, en la sentencia deberán mencionarse de forma detallada las indemnizaciones concedidas por los distintos perjuicios sufridos por la víctima».

Lo que admite el Tribunal Supremo es que de forma orientativa se pueda utilizar el Anexo introducido por la Disposición Adicional Octava de la Ley 30/1995, de 8 de noviembre, en la referida LRCSCVM (Decreto 632/1968, de 21 de marzo), cuyos módulos los actualiza la Dirección General de Seguros y Fondos de Pensiones anualmente, para así cubrir el vacío legal existente para determinar las indemnizaciones por daños derivadas de accidentes de tráfico, y ello a pesar de que se reconoce que en el sector de circulación de vehículos a motor, al igual que en el de la navegación aérea, en el de la energía nuclear o en el de los consumidores, se estructura principalmente a partir del principio de socialización del riesgo y prescindiendo de la culpa del agente causante, cosa que no ocurre en el campo laboral que estamos tratando, en el que la responsabilidad objetiva se cubre con las prestaciones de la seguridad social, pero la responsabilidad civil del empresario está basada en una responsabilidad subjetiva y culpable, lo cual se ajusta a la Directiva 89/391/CEE, respecto a la obligación del empresario de garantizar la seguridad y salud de los trabajadores.

Ahora bien, este razonamiento lleva al Tribunal Supremo a una conclusión fundamental, como es admitir que en el cálculo de la indemnización se ha de tener en cuenta la responsabilidad objetiva del empresario, ya cubierta por la prestación de la seguridad social, que compensa fundamentalmente de la pérdida de ingresos profesionales, que es lo que anteriormente se ha denominado lucro cesante. Por tanto, lo importante es identificar estos cuatro componentes básicos de la indemnización, para que así la prestación de la Seguridad Social sea descontada solamente de éste último.

Por tanto, en caso de utilizar el baremo de accidentes de tráfico, se ha de tener en cuenta que en el mismo se da un trato igualitario a los daños biológicos y psicológicos, así como a los daños morales, ya que se considera que éstos son los mismos en todas las personas, salvo prueba en contrario. Las diferencias dañosas se dan en las secuelas de la capacidad laboral, que afectan al lucro cesante, y que en el baremo se contemplan exclusivamente como factores correctores por perjuicios económicos en las Tablas IV y V.

Las reglas que establece el TS para utilizar el baremo de accidentes de tráfico y sus distintas tablas son las siguientes:

A. DAÑO CORPORAL: (lesiones físicas y psíquicas) y DAÑO MORAL (sufrimiento psíquico o espiritual).

a. Tabla I y II, en caso de Muerte.

b. Tablas III y VI, en caso de Incapacidad Permanente.

c. Tabla V, en caso de Incapacidad Temporal (compatible con otras indemnizaciones).

\section{B. DAÑO PATRIMONIAL}

- DAÑO EMERGENTE (pérdida patrimonial directamente vinculada con el hecho dañoso).-

En este caso habrá que computar los gastos concretos en los que se incurre como consecuencia del accidente. No se contempla en las Tablas y hay que probarlos. 
La considerable extensión de dichas Tablas desaconseja su inclusión en este trabajo. No obstante, para la consulta de la última actualización publicada de las mismas remitimos al lector a la Resolución de 17 de enero de 2008, de la Dirección General de Seguros y Fondos de Pensiones, por la que se da publicidad a las cuantías de las indemnizaciones por muerte, lesiones permanentes e incapacidad temporal, que resultarán de aplicar durante 2008, el sistema para valoración de los daños y perjuicios causados a las personas en accidentes de circulación (BOE número 21 de 24/01/2008).

\section{VALORACIÓN DEL DAÑO MORAL}

Nuestra propuesta de valoración del daño moral en el marco de la indemnización por accidente de trabajo parte de la identificación de los cuatro componentes básicos de la indemnización (daño extrapatrimonial, compuesto de daño corporal y daño moral y daño patrimonial, subdividido en daño emergente y lucro cesante). En este apartado nuestro objetivo es la valoración del componente relativo al daño moral. Una de las principales novedades de nuestro estudio es que aborda la cuantificación económica del daño moral adoptando la perspectiva del que lo sufre, para ello se utiliza la valoración poblacional obtenida mediante encuestas, es decir, el valor que cada persona otorga a cada año de vida libre de discapacidad y a los distintos estados de salud.

Nuestro método parte de la estimación del valor de los años de vida ajustados por calidad perdidos por el trabajador. Para ello utilizamos elementos característicos del instrumental analítico en el ámbito de la economía de la salud (años de vida ajustados por calidad, AVAC; esperanza de vida libre de discapacidad, EVLD) y en el ámbito de la economía medioambiental (valor estadístico de una vida, VEV).

El Gráfico 1 nos ofrece la secuencia de cálculo para la evaluación económica del daño moral producido como consecuencia de un accidente de trabajo.

La primera fase del cálculo permite conocer el número de AVAC perdidos por el trabajador accidentado. Para ello calcularemos la diferencia entre la Esperanza de Vida Libre de Discapacidad (EVLD) de un trabajador cualquiera del mismo sexo que el accidentado y con residencia en su misma Comunidad Autónoma y la edad del trabajador accidentado. Asumimos en este punto la equiparación operativa del concepto de AVAC con el de VLD, es decir, suponemos que los años de vida libres de discapaci-

Gráfico 1. Secuencia de cálculo para la valoración económica del daño moral

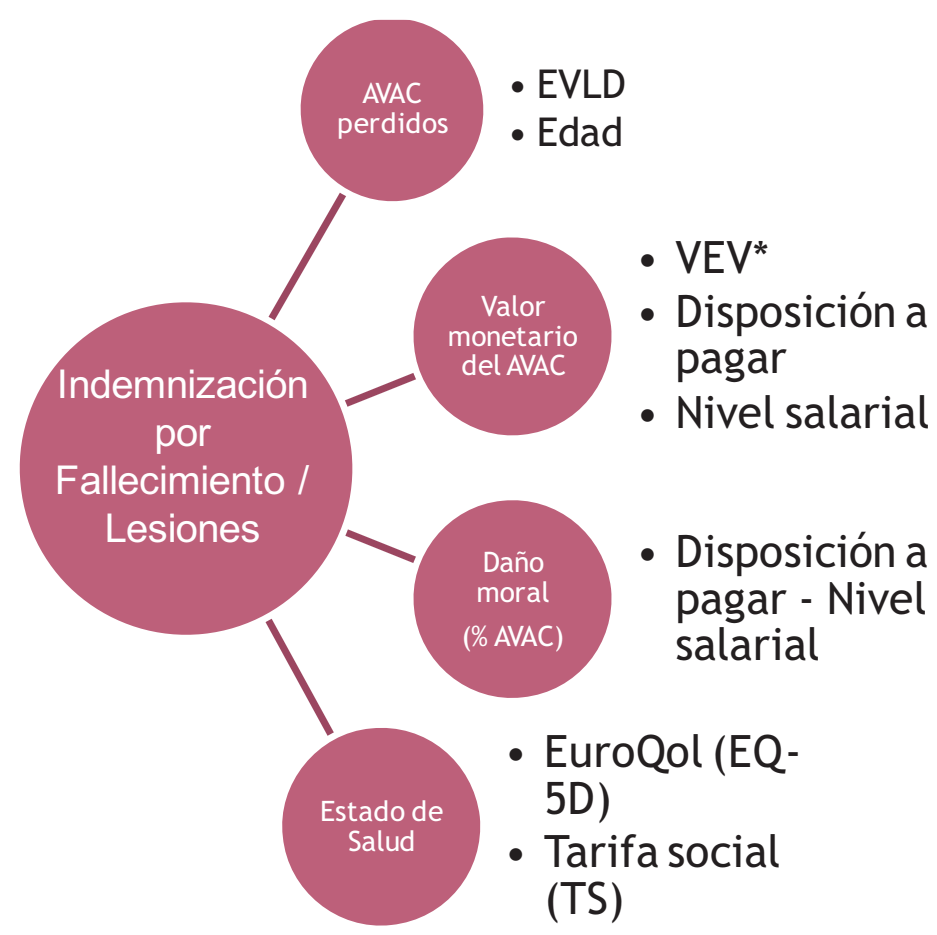


dad son años en un estado de buena salud. Aunque ambos términos presentan claras diferencias conceptuales, la utilización del concepto de EVLD como proxy de la esperanza de vida ajustada por calidad permite cubrir la laguna estadística existente dado que actualmente no disponemos de una estadística regular de esperanza de vida ajustada por calidad que nos permita conocer la esperanza de vida en términos de AVAC.

El AVAC es la unidad de medida que nos permite comparar situaciones de salud muy diversas. El AVAC es un año de vida en buena salud, esto es, es el valor del par (calidad de vida en buena salud, 1 año).

El AVAC es una medida de la salud basada en varios supuestos (Pinto, JL y Martínez, JE, 2005)(3):

1. La salud se puede reducir a dos componentes que son la calidad (Q) y la cantidad de vida (Y).

2. Cualquier estado de salud se puede representar mediante una combinación de cantidad y calidad de vida, esto es, mediante un par $(\mathrm{Q}, \mathrm{Y})$.

3. Los pacientes prefieren, en ocasiones, vivir menos años pero en mejor calidad de vida. Por tanto, los pacientes están dispuestos a ceder duración para ganar en calidad. Otros, en cambio, prefieren perder calidad para ganar cantidad. Hay, por tanto, diversas combinaciones $(\mathrm{Q}, \mathrm{Y})$ que tienen el mismo valor para las personas.

4. La salud de una persona puede medirse como la suma de la calidad de vida durante los años que dura su vida.

Afortunadamente, desde hace varias décadas la supervivencia y la edad media en el momento de la muerte siguen aumentando y, aunque más moderadamente, sigue creciendo la esperanza de vida (EV) de la población.

En este contexto los tradicionales indicadores de mortalidad se muestran cada vez más limitados para reflejar un concepto de salud acorde con objetivos del sistema sanitario, como son restaurar la salud y prevenir la enfermedad y la expectativa de la población de vivir más y mejor.

La esperanza de vida libre de discapacidad (EVLD) es un indicador muy útil para su uso poblacional porque el método de cálculo es sencillo y los resultados fáciles de interpretar. Se basa en datos de mortalidad ponderada en función de la prevalencia de discapacidad y tiene la gran ventaja de que se ha hecho un importante esfuerzo para la estandarización de los conceptos, las fuentes de información y los métodos de cálculo. Es uno de los indicadores recomendados por la Organización Mundial de la Salud para el seguimiento de las estrategias de salud para todos, y además, se ha incorporado como uno de los indicadores estructurales de la Unión Europea, de modo que estos cálculos están disponibles para los países miembros. En España contamos con una publicación reciente de Rosa Gispert et al. (2007)(4) que nos ofrece el cálculo de dicho indicador para el año 1999 ajustado por sexo y Comunidad Autónoma.

La segunda fase del proceso persigue la asignación de un valor monetario a cada año de vida ajustado por calidad.

El cálculo del valor monetario de un AVAC se puede realizar desde tres perspectivas distintas:

- A partir del consenso de expertos

- A partir de la estimación directa de la disposición a pagar por un AVAC

- A partir del concepto de Valor Estadístico de una Vida (VEV)

En España no existe un valor umbral oficialmente aceptado para la evaluación económica de nuevas tecnologías sanitarias. Dicho valor umbral se refiere al coste máximo por AVAC ganado que estaríamos dispuestos a aceptar cuando evaluamos la incorporación al catálogo nacional de prestaciones sanitarias de una nueva tecnología (equipo, medicamento, procedimiento). No obstante, al igual que en la mayoría de los países donde tampoco existe un pronunciación oficial sobre dicho valor umbral, en España existe un consenso ampliamente extendido que fija dicho valor límite en 30.000 euros.

Otra forma de calcular el valor monetario del AVAC consiste en estimarlo de forma directa a través de la disposición a pagar. Este método directo calcula el valor monetario del AVAC utilizando la fórmula siguiente:

$$
\text { VMAVAC }=\text { DP }(Z) /\left[(1-U(Z) \times t] \times p^{*}\right.
$$

Donde:

DP $(Z)$ : disposición a pagar por un tratamiento que reduce la probabilidad de tener un estado de salud $\mathrm{Z}$ no deseado. 
$\mathrm{U}(\mathrm{Z})$ : utilidad relativa que genera el estado de salud $Z$ respecto al estado buena salud

t: tiempo que permaneceremos en el estado de salud Z

p*: reducción del riesgo de quedar en el estado de salud $\mathrm{Z}$ con el tratamiento evaluado

En España encontramos un trabajo empírico de Pinto, JL y Martínez, JE (2005)(3) que emplea esta metodología. El valor monetario del AVAC obtenido por los citados autores osciló entre $9.000 €$ y 38.000 $€$. Aunque el rango de variabilidad obtenido puede considerarse excesivo, incluye el valor de $30.000 €$ habitualmente utilizado y recomendado en los trabajos de evaluación económica de tecnologías sanitarias publicados en España.

En tercer lugar, el valor monetario de los AVACs puede obtenerse a partir del valor estadístico de una vida (VEV). Dicho de otro modo, el VEV podría ser considerado como el valor presente descontado de los AVAC que un individuo espera tener durante su toda vida. En nuestro caso, este procedimiento de cómputo implica calcular previamente el VEV y después dividir este valor entre los años de vida libres de discapacidad (EVLD) estimados para una población determinada.

Este procedimiento permite obtener el valor de un AVAC a partir del VEV siempre que se utilice: a) una tasa de descuento apropiada, b) el número de años que se espera vivir y c) la calidad de vida que se espera disfrutar durante estos años. En España se han publicado recientemente dos trabajos que estiman el VEV por distintos métodos. En el trabajo de Riera Font et al. (2007)(5) ha sido estimado recientemente en 2,04 millones de euros. El cálculo del VEV se basa en el método de los salarios hedónicos. Este método parte de la estimación de una ecuación estructural de los salarios en la que se incluye como variable determinante el riesgo al que se someten los trabajadores de distintos sectores productivos, categoría y niveles salariales. El Método de Salarios Hedónicos (MSH) ha sido ampliamente utilizado desde que Thaler y Rosen (1975)(6) efectuaran la primera estimación y desarrollaran gran parte de la terminología empleada hasta el momento. Viscusi y Aldy (2003)(7) ofrecen una revisión exhaustiva de los estudios que utilizan el MSH.

En este trabajo los autores realizan una estimación del VEV a partir de una amplia base de datos del mercado de trabajo español y de la construcción de índices de riesgo laboral que incorporan simultáneamente la ocupación y la rama de actividad para el período 1997-2000. Los principales resultados de la aplicación constatan la existencia de una relación positiva entre el riesgo de accidentalidad y el salario percibido y sitúan el VEV en España en el rango de 2 a 2,7 millones de euros, a precios del 2000.

Para ilustrar este concepto, supongamos que un grupo de 100.000 personas se someten a un tratamiento preventivo que reduce la probabilidad de muerte (o de estar enfermo) en un 1 entre 100.000. El número esperado de muertes se reduce en 1 unidad dentro del grupo. Supongamos que los individuos que pertenecen a dicho grupo están dispuestos a pagar, por término medio, $20 €$ por dicha reducción del riesgo. La disposición a pagar total será $20 € \mathrm{x}$ 100.000. A esta cantidad es a lo que denominamos disposición a pagar agregada por prevenir una muerte estadística (VEV). Es importante señalar que como el VEV es igual a la disposición a pagar media dividida por la reducción del riesgo, dicho valor puede verse como la media de las relaciones marginales de sustitución entre renta y riesgo, esto es, la relación entre la reducción de la renta y la reducción del riesgo $(\Delta \mathrm{Y} / \Delta \mathrm{r})(20 / 0,000001)$.

El segundo trabajo recientemente publicado es el de Martínez Pérez et al. (2007)(8). En este trabajo los autores estiman el Valor Monetario de una Vida Estadística en España a partir de preferencias declaradas por la población general. El contexto de valoración elegido es el de los accidentes de tráfico. La metodología empleada es la Valoración Contingente. En la encuesta realizada se intentaron comunicar correctamente los riesgos mediante el recurso a ayudas visuales. Los valores obtenidos (no inferiores a 2,7 millones de euros) son similares a otras estimaciones efectuadas en Europa.

Una vez comprobada la similitud entre los tres métodos de cálculo del valor monetario del AVAC, por motivos operativos, optaremos por la tercera vía de cálculo. Esta formulación nos ofrece valores más precisos y nos permite establecer cálculos más exactos en función del sexo y el origen geográfico del trabajador accidentado.

En nuestros cálculos utilizaremos el VEV estimado en 2,04 millones de euros. Los motivos que nos llevan a tal elección son dos. Primero un criterio de prudencia valorativa nos ha llevado a elegir la estimación que ofrece los valores más bajos. En segundo lugar, la facilidad para reproducir los cálculos en el futuro es mayor en el método de los salarios hedónicos, dado que utiliza datos estadísticos y no 
requiere de un trabajo de campo específico para recoger los datos de las encuestas.

Si enlazamos la estimación del VEV con los datos sobre EVLD ofrecidos en el trabajo de Rosa Gispert podemos calcular un valor monetario para el AVAC en el entorno español. La EVLD promedio para el ámbito nacional es de 68,52 y 72,21 años para hombres y mujeres respectivamente. Por lo tanto, dividiendo los 2,04 millones de euros del VEV entre estas EVLD obtendremos el valor monetario de un AVAC, $29.772 €$ y $28.251 €$ para hombres y mujeres respectivamente.

Como podemos apreciar, para el caso español, todos los procedimientos comentados ofrecen un alto grado de concordancia situando el valor monetario del AVAC en torno a los 30.000 euros. No obstante, es preciso tener presente que dichos métodos de cálculo se basan en la disposición a pagar de los individuos por una reducción del riesgo.

Dado que la disposición a pagar por un año de vida en un estado "buena salud" mantiene una relación directa con el nivel de renta de los individuos, aunque el valor promedio se sitúe en torno a los 30.000 euros, para cada caso concreto el valor monetario del AVAC variará en función de la renta.

Para el cálculo de la indemnización por daño moral correspondiente a un trabajador concreto, el valor del AVAC debe ajustarse en proporción a la distancia existente entre el salario del trabajador accidentado y el salario promedio en su CCAA y franja etaria. Para llevar a cabo esta comparación utilizaremos la Encuesta de Estructura Salarial 2002 (9) publicada por el Instituto Nacional de Estadística con el oportuno desglose por sexos, franjas etarias y Comunidades Autónomas.

Por ejemplo, supongamos un varón de 25 años con un salario de 20.000 euros que fallece en accidente de trabajo en Andalucía. La primera cuestión sería calcular el número de AVACs perdidos por este trabajador según la diferencia entre la EVLD para un hombre en Andalucía (66,07 años) y la edad del accidentado fallecido (25 años). En este caso los AVACs perdidos son 41,07. En segundo lugar habrá que calcular el valor monetario de un AVAC para un hombre en Andalucía, dividiendo el VEV (2,04 millones de euros) entre la EVLD para los hombres en Andalucía (66,07 años). El resultado obtenido es 30.876 euros. En tercer lugar, deberemos calcular el valor del AVAC para el accidentado en función de su nivel salarial y su edad. Para ello calcularemos la proporción que existe entre el nivel salarial del acci- dentado y el estándar para los varones de esa edad en Andalucía (13.732 euros). El accidentado de nuestro ejemplo sobrepasaba el salario medio en un $45,64 \%$, luego el valor de un AVAC para este trabajador se situaría en 44.968 euros $(30.876 \times 1,4564)$.

Tal como hemos comentado anteriormente el valor monetario del AVAC se basa en la disposición a pagar por una reducción del riesgo de muerte. En esta disposición a pagar de los individuos cabe suponer que intervienen dos determinantes fundamentales, uno estaría relacionado con el nivel de renta del individuo, el otro con la valoración subjetiva que se otorga a la vida. Pasamos con este comentario a la tercera fase en la cuantificación económica del daño moral.

Si observamos los valores obtenidos para las estimaciones publicadas en España, el valor monetario del AVAC para los varones en el conjunto nacional asciende a $29.772 €$. Sin embargo, el salario promedio publicado por el INE para el año 2002 se situaba en 19.802 euros. Esto quiere decir que la disposición a pagar promedio de los individuos por un AVAC superaba en gran medida su salario medio. Ese exceso en la valoración del AVAC cabe atribuirlo al valor subjetivo de la vida. Por lo tanto, en caso de perderla, esta cantidad sería la adecuada para valorar el daño moral. En concreto, un 33,49\% del valor monetario de un AVAC refleja la valoración subjetiva del valor de la vida, por encima de aquellos aspectos relacionados con la renta del individuo. Por el contrario, un $66,51 \%$ del valor monetario del AVAC estaría relacionado con el nivel de renta y patrimonio del individuo.

Al objeto de simplificar los cálculos, consideramos adecuado utilizar en todos los casos la proporción media a nivel nacional, es decir, un $33,49 \%$, independientemente de la edad y localización geográfica del accidente.

En nuestro ejemplo anterior, el valor del daño moral para este individuo ascendería a 618.505 euros. Para obtener dicho resultado calculamos los AVAC perdidos $(66,07-25=41,07)$, los multiplicamos por el valor de un AVAC en este individuo $(44.968 €)$ y por el porcentaje del daño moral $(33,49 \%)$.

Esta forma de cálculo implica que para todos los individuos se utilizará una misma proporción para evaluar el daño moral, pero esa proporción se aplicará sobre el valor monetario del AVAC especifica de cada individuo, en función de su edad y su nivel salarial. 
Finalmente, la cuarta fase del procedimiento de cálculo propuesto recoge la adaptación del método para los casos en los que el trabajador accidentado no fallece sino que queda afectado por lesiones de carácter permanente o secuelas. En el caso de que el accidente laboral generase daños distintos a la muerte, la indemnización calculada anteriormente se reduciría en función de la tarifa social asignada al estado de salud del trabajador tras el accidente. La valoración del estado de salud se realiza mediante el indicador sintético conocido como EuroQol (EQ5D). El EQ-5D es un instrumento simple, genérico, internacional y estandarizado, diseñado para describir y valorar la calidad de vida relacionada con la salud.

El EQ-5D es una medida genérica simple que incluye sólo 5 dimensiones básicas:

\section{Movilidad}

\section{Cuidado personal}

\section{Actividades cotidianas}

\section{Dolor/malestar}

\section{Ansiedad/depresión}

Contempla 3 niveles para cada dimensión, de modo que proporciona un perfil descriptivo y una valoración del propio estado de salud. Cada estado de salud está definido por 5 dimensiones. Cada dimensión tiene 3 niveles, generando un total de 243 estados de salud teóricamente posibles. Los 3 niveles reflejan grados crecientes de gravedad, es decir, ningún problema (nivel 1), algunos o moderados problemas (nivel 2), y muchos problemas (nivel 3). De esta forma, cada estado de salud queda definido por un número de 5 dígitos. Por ejemplo, el estado 11111 indica que la persona no tiene problemas en ninguna de las 5 dimensiones.

El EQ-5D permite asignar valores a todos los estados de salud, obteniendo un índice para cada uno de los 243 estados de salud posible, así como para los estados "inconsciente" y "muerte". El resultado es una tarifa o sistema de ponderación que refleja las preferencias de distintas poblaciones para cada uno de los estados de salud generados por el EQ-5D. Las tarifas obtenidas a partir de la valoración de los estados de salud por la población general se llaman tarifas sociales.

En España se disponen de dos tarifas que muestran las preferencias de la población general hacia los estados de salud, una calculada mediante el método de la Escala Visual Analógica (EVA) y la otra calculada mediante el método de la Equivalencia Temporal (ET). Ambas se obtuvieron a partir de la valoración revelada por una muestra aleatoriamente seleccionada y representativa del conjunto de la población. Siguiendo las recomendaciones de la literatura especializada, en nuestro caso proponemos la utilización de la tarifa social basada en la Equivalencia Temporal. La equivalencia temporal es una técnica que contempla el estado de salud como un concepto que abarca periodos de tiempo, más que el estado de salud en un punto del tiempo. Está ideada para hacer intercambios de estados de salud (unos peores por otros mejores) entre periodos de tiempo. La elaboración de la tarifa social en nuestro país fue realizada por Xavier Badia, como coordinador del Grupo EQ-5D en España (10)(11).

El rango de valores en que se mueve la tarifa varía entre -1 y 1 , asignando a los estados de "salud perfecta" (11111) y "muerte" los valores 1 y 0 respectivamente. No obstante, el estado de salud con problemas graves en todas las dimensiones (33333) obtiene un valor inferior al del estado "muerte" 0,6533. Asimismo, el estado "inconsciente" también presenta un valor negativo igual a $-0,5085$.

Para el cálculo del daño moral en trabajadores accidentados con resultado de lesiones en distintos niveles aplicaremos el instrumento EQ-5D, lo que nos permitirá determinar su estado de salud tras el accidente entre los 243 estados de salud posibles. A su vez, el estado de salud nos llevará a conocer la tarifa social otorgada al mismo, es decir, la preferencia que la población en general muestra por ese estado de salud en una escala que va desde 1 hasta -1 .

Una vez conocido el valor de la tarifa social (TaS), ponderaremos el valor monetario de los AVACs perdidos por el trabajador con el valor resultante de la diferencia entre 1 (tarifa social para el estado de salud perfecta) y el valor de la tarifa social asignada al estado de salud del trabajador (1-TaS). Por ejemplo, si un estado de salud tiene una tarifa social igual a 0,20 esto indica que dicho estado es valorado como el $20 \%$ de un estado de "salud perfecta", luego el trabajador que queda en este estado de salud como consecuencia del accidente perdería el $80 \%$ de los AVACs que teóricamente le quedaban por vivir. Dado que el trabajador no ha fallecido, el daño moral no contempla la pérdida del $100 \%$ de los AVACs sino, en este caso, de un $80 \%$ de los mismos. No obstante, en algunos casos, cuando la tarifa social es menor que cero, la ponderación de los AVACs perdidos puede ser superior al $100 \%$, de modo que el trabajador recibiría una indemnización 
en concepto del daño moral sufrido superior a la que recibiría en caso de muerte.

De acuerdo con Pintos Ager (12), consideramos que el objetivo de la reparación íntegra del daño es un buen criterio parcial dentro del derecho de daños, pero no sirve como objetivo central de este sector del ordenamiento, ni para su finalidad preventiva ni para la compensatoria. En particular, vale como criterio para la reparación del daño patrimonial, pero no es aplicable al daño moral.

\section{CONCLUSIONES}

En el escenario internacional los sistemas de indemnización por accidentes de trabajo ofrecen una notable heterogeneidad que no induce a esperar un eventual proceso de convergencia hacia un sistema único (13). Incluso dentro de la Unión Europea el reducido impacto de dicha heterogeneidad sobre los objetivos fundamentales del mercado único (no discriminación, libre circulación de trabajadores, defensa de la competencia, etc.) no ejercen la necesaria presión para forzar un proceso unificador de la legislación comunitaria en esta materia. Por lo tanto, en principio, parece oportuno acometer reformas en el terreno legislativo y jurisprudencial sin temor a que éstas sean absorbidas por un posible proceso unificador.

En España, dos recientes sentencias del Tribunal Supremo han abierto un nuevo escenario en el que no se exonera al empresario del deber de reparar los perjuicios causados en un accidente de trabajo o enfermedad profesional por el incumplimientos de sus obligaciones en materia de seguridad, al mismo tiempo que se permite utilizar para el cálculo de la indemnización el baremo de tráfico u otros métodos.

Tradicionalmente se ha mantenido el criterio de utilizar los baremos en el cálculo de las indemnizaciones de daños corporales. El principal efecto indeseado de la evaluación abierta es la variabilidad en las indemnizaciones (12). La asociación entre reparación integral y arbitrio sin instrumentos produce arbitrariedad, de modo que la cuantía de las indemnizaciones varía casi erráticamente. Por lo tanto, la defensa de los baremos se ha basado fundamentalmente en la necesidad de objetivar los criterios de valoración, evitando de este modo la tan temida arbitrariedad y la dispersión en los montantes indemnizatorios calculados en cada caso. Sin embargo, la defensa de los baremos en la mayoría de los países, si bien como elemento orientativo, no ha servido para hacer converger a nivel internacional los importes indemnizatorios ante circunstancias similares.
La jurisprudencia española, en línea con la legislación italiana, ha planteado la necesidad de delimitar claramente los distintos conceptos indemnizatorios comprendidos en el montante global, haciendo un especial hincapié en la necesidad de valorar de forma específica el denominado daño moral.

La cuantificación monetaria del daño moral no es una materia ajena a la discusión y a la controversia. En determinados ámbitos resulta chocante poner precio a los sentimientos morales y a la vida misma de las personas. No obstante, los continuos avances de la economía, y en concreto de la economía de la salud, han permitido el desarrollo de numerosos instrumentos conceptuales válidos y aceptados en el terreno de la evaluación de tecnologías sanitarias o en la evaluación de las políticas preventivas, ya sea en el terreno laboral o en el medioambiental.

El método que hemos presentado en este trabajo se basa en la utilización de este instrumental analítico bien conocido en otras áreas de conocimiento. La cuantificación económica del daño moral sufrido se ha concretado en el 33,49\% del valor monetario asignado a los AVACs perdidos como consecuencia del accidente. El cálculo de la indemnización propuesta como compensación del daño moral resulta de la siguiente secuencia de cálculos:

1. AVACs perdidos $=E V L D-$ Edad del fallecido

2. Valor monetario estándar del AVAC: VEV / EVLD

3. Valor monetario personalizado del AVAC $=$ VMS AVAC x Factor corrector (salario / salario promedio)

4. EUROQOL_5D: determinación del estado de salud

5. Tarifa Social: $-1 \leq \mathrm{TaS} \leq 1$

6. Indemnización por daño moral=AVACs perdidos x (1-TaS) x VMP_AVAC x 0.3349

La valoración económica del daño moral calculada en este trabajo incorpora algunas hipótesis restrictivas que pudieran ser interpretadas como limitaciones del mismo. Sin embargo, dichas restric- 
ciones han sido mantenidas de forma expresa en pro de la claridad expositiva de la metodología propuesta. Nos referimos a dos supuestos que asumimos en nuestra secuencia de cálculo:

a) El daño moral sufrido por el trabajador es de carácter permanente o definitivo

b) Suponemos que el trabajador accidentado gozaba de "buena salud" en el momento de sufrir el accidente

La supresión de ambas restricciones puede ser incorporada perfectamente en nuestro esquema de cálculo. En el primer caso bastaría con traducir a AVACs perdidos el daño moral sufrido durante un tiempo determinado por el trabajador accidentado. Respecto a la segunda restricción, el problema se resolvería del mismo modo, en el momento de calcular los AVACs perdidos por el trabajador habría que tener en cuenta la tarifa social de su estado de salud previo. Un trabajador con un estado de salud previo inferior a la unidad vería reducido el número de AVACs perdidos en una proporción igual a 1$\mathrm{TaS}_{\mathrm{i}}$, siendo $\mathrm{TaS}_{\mathrm{i}}$ la tarifa social de su estado de salud inicial.

Tradicionalmente la forma de evitar la arbitrariedad en la determinación judicial de la indemnización por accidentes de trabajo ha llevado a distintos autores a la defensa de los baremos. Sin embargo, consideramos que la metodología propuesta basada en el concepto de AVAC y su cuantificación monetaria ofrece claras ventajas respecto al método de cálculo basado en baremos:

a) Permite la personalización de los cálculos teniendo en cuenta las características personales y laborales del trabajador accidentado. Dicha personalización tiene en cuenta las variables relativas a la edad, la Comunidad Autónoma de residencia, el sexo, y las expectativas profesionales del trabajador a través de su nivel salarial.

b) Incorpora un criterio de equidad según el cual se asume que todas las personas valoran subjetivamente su vida en la misma cuantía. Este criterio se hace operativo a través del porcentaje promedio en el que el valor monetario de un AVAC excede al salario medio para el conjunto de la población (daño moral: 33,49\% AVAC). c) Incorpora dos innovaciones metodológica relevantes:

a. Perspectiva social de la medida: la utilización del baremo de tráfico implica la utilización del criterio de las compañías de seguros que deben hacer frente a las indemnizaciones. El cálculo directo basado en AVAC se basa, por el contrario, en el valor social que la población en general asigna a cada estado de salud, de modo que la perspectiva de valoración utilizada es una perspectiva social.

b. Criterios explícitos y transparentes: los criterios utilizados para la elaboración de los baremos suelen incorporar cálculos actuariales y otras consideraciones que en muy extrañas ocasiones se dan a conocer. El método de cálculo directo que proponemos ofrece una transparencia absoluta en cuanto a las variables utilizadas y a sus fuentes. Dicha transparencia lo dota de una manifiesta neutralidad, de modo que la posible dispersión entre casos queda claramente explicada por la propia secuencia de cálculo.

d) La posibilidad de llevar a cabo actualizaciones queda totalmente garantizada con un coste mínimo de actualización de las tablas utilizadas. Esta puede considerarse la principal ventaja del método propuesto, esta forma de cálculo permite que las cuantías indemnizatorias por daño moral estén constantemente actualizadas incorporando de forma inmediata los cambios sociales, económicos y demográficos. Los cambios en la estructura salarial, la convergencia regional, la esperanza de vida libre de discapacidad y, por supuesto, el valor estadístico de la vida reflejan el carácter dinámico de una sociedad moderna donde la protección al trabajador debe mantenerse en consonancia con dichas evoluciones.

Las ventajas comentadas sitúan el método de cálculo directo como una alternativa que mejora las posibilidades que ofrece la utilización del baremo de tráfico para la cuantificación económica de las indemnizaciones por accidentes de trabajo. 


\section{BIBLIOGRAFÍA}

(1) López García de la Serrana, J. (dir.) Manual de Valoración del Daño Corporal. Guía de aplicación del sistema de baremación para accidentes de circulación. Pamplona (Navarra).Thomson, Aranzadi; 2007.

(2) Álvarez Vigaray, R. La responsabilidad por daño moral. Anuario de Derecho Civil. 1966; 19 (1): 81-116.

(3) Pinto Prades, J.L. y Martínez Pérez, J.E. Criterios prácticos para el cálculo de las indemnizaciones. Algunos ejemplos reales, Ekonomiaz, 2005; 1(60): 193-209.

(4) Gispert R, Ruíz-Ramos M, Arán Barés, M et al. Diferencias en la esperanza de vida libre de discapacidad por sexo y Comunidades Autónomas en España. Rev Esp Salud Pública 2007; 81: 155-165.

(5) Riera Font A, Ripoll Penalva AM y Mateu Sbert J. Estimación del valor estadístico de la vida en España: Una aplicación del Método de Salarios Hedónicos. Hacienda Pública Española / Revista de Economía Pública, 2007; 181(2/2007): 29-48.

(6) Thaler R y Rosen S. The Value of Saving a Life: Evidence from the Labor Market, en N. Terleckyj (ed.), Household Production and Consumption, Cambridge, MA: Columbia University Press; 1975:, 265-300.
(7) Viscusi WK y Aldy JE. The Value of Statistical Life: a Critical Review of Market Estimates throughout the World. Journal of Risk and Uncertainty 2003; 27 (1): 5-76.

(8) Martinez Pérez JE, Abellán Perpiñán JM y Pinto Prades, JL. El valor monetario de la vida estadística en España a través de las preferencias declaradas. Hacienda Pública Española / Revista de Economía Pública, 2007; 183(4/2007): 125-144.

(9) Instituto Nacional de Estadística. Encuesta de Estructura Salarial 2002. Resultados definitivos. 2004. Consulta a INEBase en diciembre de 2007.

(10) Badía X, Roset M, Montserrat S, et al. La versión española del EuroQol: Descripción y aplicaciones. Med Clin 1999; 112 (Supl 1): 79-86.

(11) Badía X. y De Charro, F. EuroQol, un instrumento para valorar la salud. EQ 5- D guía del usuario, versión española. Med Clin 1999; 114: 6-14.

(12) Pintos Ager, J. Baremos. InDret Revista para el Análisis del Derecho 2000; 1

(13) Parsons, C. Liability Rules, Compensation Systems and Safety at Work in Europe. The Geneva Papers 2002; 27(3): 358-382. 